DOI: http://dx.doi.org/10.22198/rys.2018.72.a378

Artículos

\title{
Efectos esperados de la extracción de gas shale en el noreste de México: un enfoque cualitativo
}

\author{
Expected effects of shale gas extraction \\ in northeastern Mexico: a qualitative approach
}

\author{
Dionicio Morales Ramírez* \\ Ruth Roux ${ }^{* *}$
}

Resumen: en este artículo se identificaron y valoraron los efectos esperados de la extracción de gas shale en Tamaulipas, Nuevo León y Coahuila, ante la llegada inminente de compañías nuevas a la cuenca de Burgos, en el noreste de México. Con la metodología de criterios relevantes integrados se recopilaron datos de informantes clave de los sectores académico, gubernamental y empresarial, de los tres estados. Según los resultados, 65 por ciento de las variables empleadas para evaluar dichos efectos, derivados del desarrollo de la industria en la región, se asocian con cambios positivos, 15 con negativos y 20 se mantienen inalterables. Se sobreestimaron los beneficios esperados por la explotación de gas oil/shale, y existe preocupación por la disponibilidad de agua, dadas

\footnotetext{
* Autor para correspondencia: Facultad de Ingeniería "Arturo Narro Siller" de la Universidad Autónoma de Tamaulipas. Centro Universitario Tampico-Madero, C. P. 89140. Ciudad Tampico, Tamaulipas, México. Teléfono: (833) 241 2000, extensión 3475. Correo electrónico: dmorales@docentes.uat.edu.mx

** Centro de Investigaciones Sociales de la Universidad Autónoma de Tamaulipas. Centro de Gestión del Conocimiento, sexto piso, Centro Universitario Adolfo López Mateos, C. P. 87149 , Ciudad Victoria, Tamaulipas, México. Correo electrónico: rrouxr@gmail.com
} 
las condiciones geográficas y climatológicas de la región. Aunque la metodología empleada no especifica que se deba aplicar a un número óptimo de actores, se reconoce que el análisis podría mejorar significativamente entre mayor sea la cantidad de participantes. La contribución principal al campo de la evaluación de las repercusiones sociales es la adaptación de esta metodología, debido a la escasa literatura sobre el tema.

Palabras clave: evaluación de impacto; opinión pública; fracturación hidráulica; suministro de gas; criterios relevantes integrados; noreste de México.

Abstract: this article identifies and assesses the expected effects of shale gas extraction in Tamaulipas, Nuevo Leon and Coahuila in the face of the imminent arrival of new companies to Burgos Basin in northeastern Mexico. Using the integrated relevant criteria methodology, data of key informants from the academic, governmental and business sectors in the three states were collected. According to the results, 65 percent of the variables used for assessing these effects, as a consequence of the development of industry in the region, are associated with positive changes, 15 percent with negative changes and 20 percent remain unchanged. Expected benefits from shale gas/oil exploitation were overestimated; moreover, there is concern about the availability of water, given the geographical and climatological conditions of the region. Although the methodology used does not specify it must be applied to an optimal number of actors, it is to be admitted that the analysis could be significantly improved the greater the number of participants is. The main contribution to the field of assessment of social repercussions is that of having adapted this methodology because of the existing scarce literature on the subject.

Keywords: impact assessment; public opinion; hydraulic fracturing; gas supply; integrated relevant criteria; northeastern Mexico. 
Recibido el 3 de febrero de 2016. Aceptado el 27 de marzo de 2017.

\section{Introducción}

La reforma energética aprobada en México, en 2013, abre legalmente el mercado del sector a la iniciativa privada. Ante esta situación, parece ser cuestión de tiempo que empresas dedicadas a la explotación de hidrocarburos lleguen al noreste del país. Lo preocupante es que el desarrollo de este tipo de actividades, como la extracción de gas shale, mediante la técnica del fracking, ha provocado muchos problemas sociales y ambientales en Estados Unidos, Canadá, Francia e Inglaterra, lo que señala que el costo social a largo plazo puede ser más alto que los beneficios económicos que se puedan obtener en el corto, por la explotación de estos recursos naturales. Por ello es necesario realizar estudios que ayuden a identificar los posibles cambios en los ámbitos social y ambiental.

En este sentido, en la nueva Ley de Hidrocarburos se menciona la necesidad de analizar el impacto social antes de dar una asignación o publicar convocatorias para licitar contratos para la exploración o extracción de hidrocarburos, para entender mejor los cambios sociales que se pudieran experimentar en el área (Diario Oficial de la Federación, DOF 2014). Lo anterior permitirá obtener información valiosa, que ayude a los gobiernos, las comunidades y los desarrolladores de la industria a identificar los posibles problemas sociales asociados al proyecto, e implementar programas y acciones específicas para evitarlos o minimizarlos (Burge y Vanclay 1996). De acuerdo con Horton y Leslie (1955), un problema social es "una condición que afecta a un número significativamente considerable de personas, de un modo considerado inconveniente y que según se cree debe corregirse mediante la acción social colectiva" (citado en Suárez 1989, 1).

Debido a lo anterior, en México, los estudios de impacto con énfasis en las cuestiones sociales están tomando mayor relevancia, como los métodos de evaluación asociados con el desarrollo de proyectos, programas y políticas que inciden en la dinámica económica y social de las localidades involucradas. Y aunque no hay una 
fórmula exacta para elaborar este tipo de evaluación, se han identificado algunos procedimientos, como el análisis de interesados, la elaboración de encuestas, entrevistas y aplicación de metodologías como los criterios relevantes integrados para la valoración de los impactos. También el Interorganizational Committe on Guidelines and Principles for Social Impact Assesment (2005) propuso los pasos siguientes: a) participación pública; b) identificación de alternativas; c) línea base; d) alcance; e) proyección de los efectos estimados; f) respuestas a la predicción de impactos; g) impactos indirectos y acumulativos; h) cambios en alternativas; i) mitigación y j) monitoreo. La International Finance Corporation del World Bank Group presenta una guía similar para realizar estudios de impacto ambiental y social. ${ }^{1}$

En este trabajo se elaboró el paso cuatro de la metodología propuesta por la Interorganizational Committe on Guidelines and Principles for Social Impact Assesment, en el cual se identifican los efectos probables, y se construye un índice de valoración de ellos, a partir de la revisión de la literatura. Para hacerlo se aplicó un instrumento a un grupo de especialistas en el tema para que calificaran, desde su perspectiva, las repercusiones que tendrían las actividades de explotación de gas shale en la región, en la migración, las oportunidades laborales, los ingresos económicos, la infraestructura del lugar y los problemas sociales. Esa calificación permite identificar dichas repercusiones y clasificarlas de acuerdo con su significancia o prioridad.

Los resultados presentados aquí forman parte del proyecto "Diagnóstico y análisis del impacto social de la exploración y explotación de gas/oil shale, relacionado con la cultura, la legalidad, los servicios públicos y la participación de los actores sociales en los estados de Coahuila, Nuevo León y Tamaulipas", número 38245838, financiado por el Fondo Institucional de Fomento para el Desarrollo Científico, Tecnológico y de Innovación del Consejo Nacional de Ciencia y Tecnología.

1 Véase International Finance Corporation: http://www.ifc.org/wps/wcm/connect/296ae9 80488551f5aa0cfa6a6515bb18/ESIA.pdf?MOD=AJPERES 


\section{Beneficios económicos y costos sociales de la extracción de gas shale}

La apertura comercial del sector energético en México puede ser buena en términos económicos, pues incentiva la explotación de los recursos naturales de manera eficiente, ante la incapacidad aparente del Estado para administrarlos y, sobre todo, para estimular su crecimiento, aunque tampoco hay una garantía de que las empresas privadas lo hagan bien. Como Flores $(2014,3)$ señala "es posible encontrar ejemplos de empresas estatales eficientes y empresas privadas ineficientes. Sin embargo, los costos y riesgos que implica la operación ineficiente de una empresa privada recaen sobre los accionistas, mientras que en las empresas del Estado recaen sobre los contribuyentes". En este mismo sentido, la apertura del mercado le permite al gobierno federal liberar recursos y destinarlos a otros sectores, y así obtener rentas económicas y, en el caso del gas natural, el país podría ser autosuficiente, y dejar de importarlo. De acuerdo con la Secretaría de Energía (2014), en 2013 se importaron 2516.6 millones de pies cúbicos por día (MMpcd) de gas natural, es decir 18.1 por ciento más que el año anterior. Además, la llegada de empresas incrementa las oportunidades laborales para los residentes de la región y aumenta las actividades económicas directas e indirectas.

Sin embargo, el desarrollo de este tipo de industria genera varios problemas locales, estudiados ampliamente en la literatura de las ciudades boom (energy boomtowns en Estados Unidos). Por ejemplo, según Kohrs (1974), en las ciudades donde se explotan hidrocarburos han aumentado los problemas mentales, crímenes, divorcios, suicidios y alcoholismo, en comparación con las que no se dedican a ello. Por lo que será necesario crear terapias psicológicas para superarlos, así como aumentar las instalaciones y los programas recreativos para hombres y mujeres de todas las edades tomando en cuenta sus necesidades. Entonces, el objetivo de la comunidad es alcanzar el bienestar psicológico de todos, y no sólo de quienes no sufren crisis humanas debido a la riqueza alcanzada.

Davidson (1979) señala que dichos impactos dependerán, en gran medida, del tiempo de residencia de sus habitantes, es decir, son diferentes entre la gente que ha vivido en el lugar antes del desarrollo de 
la industria y la que ha llegado a raíz del establecimiento de ella. Por ejemplo, es común que los trabajadores y sus familias recién llegados experimenten condiciones deficientes en la calidad de las viviendas, servicios públicos y médicos, así como estrés por mudarse a una comunidad extraña y aislada, la cual puede rechazarlos y causarles un posible aislamiento social. Los residentes de la localidad suelen experimentar mayor estrés por el aumento de tráfico vehicular y el ruido, comienzan a modificar sus hábitos y costumbres como el de cerrar siempre la puerta principal del hogar, o dejar de caminar a solas en lugares y en horarios en los que antes lo hacían (Jacquet 2009).

Las características descritas fueron moldeando lo que hoy se conoce como el boomtown model o disruption model, y que ha sido la base para describir el crecimiento rápido de las localidades, atribuido al desarrollo industrial, así como los cambios en las relaciones sociales, la cohesión comunitaria, la salud física y mental, los derechos básicos en el sistema político, los temores y aspiraciones, así como el medio ambiente de la comunidad (Vanclay 2003). A pesar de que el tema se ha estudiado desde los años setenta, la evidencia sobre los efectos económicos es variada, porque los hay positivos y negativos. Pero en el ámbito social se han encontrado principalmente los negativos (Markussen 1978; Freudenburg 1984; Merrifield 1984; Seyfrit 1988; Perdue et al. 1999, citados en Jacquet 2009).

Hay estudios para determinar cómo percibe la gente las actividades extractivistas, aunque su análisis ha sido más descriptivo que predictivo. Así, Brown et al. (2005) mencionan que durante las primeras etapas, la percepción negativa de las comunidades es muy alta, en comparación con las posteriores; es posible que esto se deba a la incertidumbre y a la falta de información de la gente. Shaft et al. (2013) señalan que existe una asociación positiva muy fuerte entre las percepciones de riesgo y oportunidad, asociadas con el tema de la extracción de gas, y que la intensidad de éstas se relaciona con la cantidad de pozos perforados. Según Theodori (2012), hay más percepciones negativas en los residentes de los estados en donde la industria se encuentra más desarrollada, con respecto a aquéllos en donde no lo está. Theodori y Jackson-Smith (2010) mencionan que la población en general desconfía de la intrusión de la industria del gas, y está disgustada debido a la problemática social y medioambiental que se 
genera alrededor de ella, aunque reconoce los beneficios económicos asociados con las actividades de ésta.

Un caso de estudio emblemático, sobre impactos sociales de la extracción de gas, es el de la cuenca de lutitas Marcellus Shale, en Estados Unidos, que se extiende por Virginia Occidental, Ohio, Pensilvania y Nueva York, que algunos geólogos la consideran como la más grande del mundo. En 2013 su producción de gas fue de 8 mil MMpcd en promedio ${ }^{2}$ (Barbosa 2014). Dentro de esta cuenca se sitúa Pensilvania, denominada como el epicentro de perforación de Estados Unidos, que ocupa más de una tercera parte de ella. El rápido desarrollo de las actividades de explotación, mediante la fracturación hidráulica -fracking- en este lugar, ha provocado altos costos sociales en las áreas rurales. La evidencia empírica encontrada ahí señala incremento en los accidentes viales, arrestos civiles por disturbio, infecciones de trasmisión sexual, aumento del crimen y el desorden, y la consecuencia de eso es un descenso en la calidad de vida (Food and Water Watch 2013). Esto indica los altos costos sociales locales y regionales de la perforación y explotación de hidrocarburos, en específico del gas shale, mediante el fracking.

Según Jacobsen y Parker (2014), en las localidades del occidente de Estados Unidos, en donde se ha extraído gas y petróleo, el ingreso per cápita ha aumentado hasta 10 por ciento en el corto plazo. Aunque la situación no es la misma en un periodo más largo, ya que la caída aproximada del ingreso es de 6 por ciento más si no se hubiera explotado el recurso. Por ello, los autores concluyen que la explotación de gas y petróleo para las comunidades locales es una maldición en términos de ingresos per cápita.

Por otro lado, Muller y Muller (2013) señalan que la extracción de gas oil/shale no es del todo mala, debido a que contribuye a la reducción de emisiones de gases de efecto invernadero, o las partículas de suspensión de menos de 2.5 micras de contaminación, conocidas como PM2.5, y que causan aproximadamente tres millones de muertes cada año, sobre todo en los países en desarrollo. Agregan que los efectos negativos para el medio ambiente, como la gran cantidad de

2 Reporte de Russell Gold, en The Wall Street Journal (2008). https://www.wsj.com/articles/ SB120709326316581793 


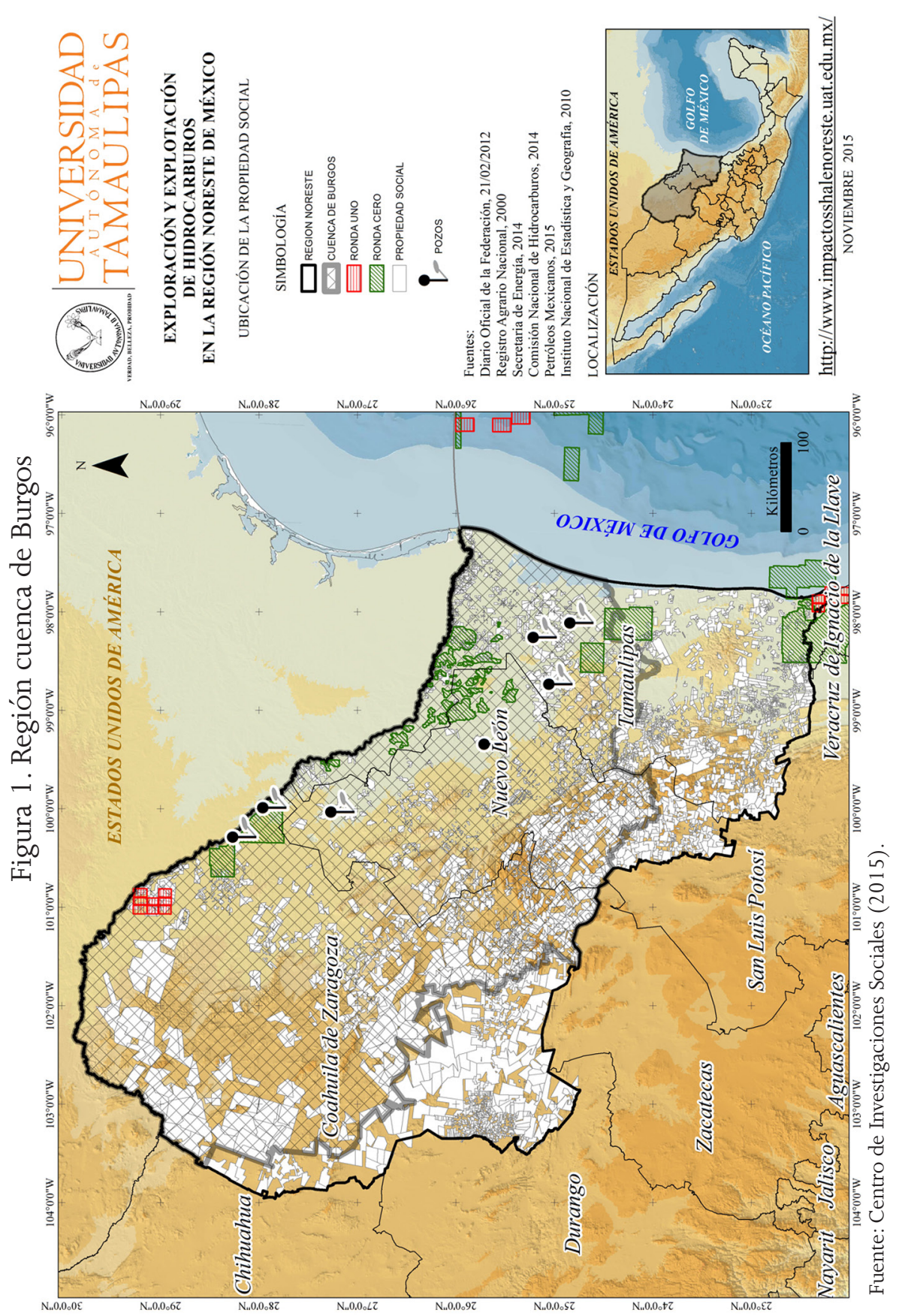


agua que requiere, las fugas de metano o la contaminación al subsuelo son falsas o, en todo caso, se pueden controlar mediante una regulación estricta; de manera que se debería tomar más en serio la explotación de este tipo de energético.

De acuerdo con un reporte emitido por la Energy International Administration $(2013,6)$, México tiene un gran potencial para la extracción de gas shale (lutitas), y la región noreste juega un papel vital, porque ahí está la cuenca de Burgos, que aporta 72 por ciento de este tipo de energético. El recurso recuperable técnicamente es de 545 billones de pies cúbicos de gas shale y de 13 mil millones de barriles de crudo no convencional, y esto coloca a México como uno de los destinos con mayor atractivo para las inversiones en el ramo (Barrueta 2015).

La cuenca de Burgos, localizada en el noreste del país, comprende 31 municipios de Coahuila, 48 de Nuevo León y 19 de Tamaulipas, que a raíz de la reforma energética, aprobada en 2013, han incrementado su importancia para la atracción de inversión privada, por la gran cantidad de reservas estimadas de gas no convencional que tienen (Secretaría de Medio Ambiente y Recursos Naturales, SEMARNAT 2008) (véase Figura 1).

Por lo anterior, se deben elaborar estudios que ayuden a valorar las repercusiones económicas, ambientales y sociales que genera la explotación para la zona. Ello permitirá que las administraciones locales y regionales se preparen lo mejor posible para afrontar la problemática asociada con ella. Este análisis se centra en los efectos cualitativos.

\section{Metodología para evaluación de efectos}

Aquí se emplea la metodología denominada criterios relevantes integrados, propuesta por Buroz (1994), que por lo común se utiliza para elaborar índices de impacto ambiental, pero se amplió para analizar las variables de interés, aunque uno de sus subcomponentes está relacionado directamente con los efectos socioeconómicos. El objetivo de dicha metodología es valorar la "significancia" en las variables, para lo que se usa la matriz de doble entrada, conocida como de Leopold (Buroz 1994), en la cual se identifican los impactos como positivos, 
neutrales y negativos, y después se califica su severidad, extensión territorial, duración en el tiempo, capacidad para volver al estado inicial "reversibilidad" y probabilidad de ocurrencia. Los resultados permiten construir un índice de valoración o de impacto sobre cada variable y visualizar, desde la perspectiva de los informantes clave (especialistas o gente relacionada con el tema), los efectos que se podrían esperar en la región ante la explotación de los hidrocarburos y del gas shale.

\section{La muestra de expertos}

La metodología original recomienda trabajar con un grupo multidisciplinario, para evaluar los impactos ambientales requeridos para la ejecución del estudio del proyecto, por ejemplo: un biólogo, sociólogo, arqueólogo, geólogo, ambientalista, eléctrico y a un mecánico, entre otros. Aquí se eligió a tres especialistas que representaran las posturas académica, gubernamental y empresarial cuyo puesto fuera clave, que estuvieran relacionados de alguna manera con el tema, y dentro del área de estudio. En todo momento se buscó que las personas que contestaran el instrumento tuvieran el mismo puesto en los tres estados pero, por falta de respuesta del informante o una estructura organizacional diferente entre ellos, la muestra quedó estructurada como se describe a continuación. En Tamaulipas se trabajó con la directora de un centro universitario en donde se imparten carreras relacionadas con el sector energético, por tanto se espera que esté informada y mantenga una visión de mediano y largo plazo sobre la industria, y cómo repercutirá en la oferta académica de la universidad. En Nuevo León, el profesor desarrolló su tesis de doctorado en el sector energético, por lo que tiene un panorama amplio y especializado sobre el tema. En Coahuila, el académico es el director de proyectos en la Corporación Mexicana de Investigación en Materiales, S.A. de C.V., un centro público de investigación perteneciente al Consejo Nacional de Ciencia y Tecnología, donde ha participado como gerente de aseguramiento de calidad y director de planeación y director de estudios estratégicos.

En el sector gubernamental se buscó el acercamiento con los directores de las secretarías de Energía o dependencias que tuvieran 
que ver con el tema. En Tamaulipas, la Agencia Estatal de Energía es la encargada de coordinar y dar seguimiento a la agenda energética del estado. Sin embargo, por cuestiones de tiempo, quien colaboró fue el coordinador de estudios y proyectos técnicos. En Nuevo León y Coahuila, al momento de aplicar el instrumento, las secretarías no tenían un titular; en Nuevo León el encargado de energía dependía del director de la Secretaría de Desarrollo Económico, por lo que se contactó a la directora en turno. En Coahuila no se identificó a alguien que fungiera como representante de energía, por lo que se buscó al secretario de Desarrollo Económico, pero no se obtuvo respuesta favorable, además se contactó a la diputada encargada de los asuntos energéticos pero, a pesar de los esfuerzos, no se tuvo respuesta. ${ }^{3} \mathrm{La}$ misma dinámica se llevó a cabo con el sector empresarial, en donde sí fue posible establecer contacto con los representantes de las diversas cámaras (véase Figura 2).

Figura 2. Grupo multidisciplinario

\begin{tabular}{|c|c|c|c|}
\hline Estado & Institución & Nombre & Puesto \\
\hline \multirow{3}{*}{ Tamaulipas } & $\begin{array}{c}\text { Universidad Autónoma } \\
\text { de Tamaulipas }\end{array}$ & $\begin{array}{c}\text { Doctora } \\
\text { Rosa Issel } \\
\text { Acosta González }\end{array}$ & $\begin{array}{c}\text { Directora } \\
\text { Unidad Académica } \\
\text { Reynosa-Aztlán }\end{array}$ \\
\hline & $\begin{array}{l}\text { Agencia Estatal } \\
\text { de Energía }\end{array}$ & - & - \\
\hline & $\begin{array}{c}\text { Cámara Nacional } \\
\text { de Comercio }\end{array}$ & ---- & ------- \\
\hline \multirow{3}{*}{ Nuevo León } & $\begin{array}{c}\text { Instituto Tecnológico } \\
\text { de Estudios Superiores } \\
\text { de Monterrey-campus } \\
\text { Monterrey }\end{array}$ & $\begin{array}{c}\text { Doctor } \\
\text { Fabián } \\
\text { Pino Pérez }\end{array}$ & Profesor-investigador \\
\hline & $\begin{array}{c}\text { Secretaría de } \\
\text { Desarrollo Económico }\end{array}$ & ----- & ---- \\
\hline & $\begin{array}{c}\text { Cámara de la Industria } \\
\text { de Transformación, } \\
\text { zona oriente }\end{array}$ & --- & --- \\
\hline
\end{tabular}

3 La idea inicial era identificar la postura de los actores en puestos clave, y relacionados con el tema energético dentro del sector gubernamental, empresarial y académico. Se reconoce que incluir también el punto de vista de la sociedad civil enriquecería de manera sustancial el trabajo. 


\begin{tabular}{|c|c|c|c|}
\hline \multirow{7}{*}{ Coahuila } & $\begin{array}{c}\text { Corporación Mexicana } \\
\text { de Investigación } \\
\text { en Materiales, } \\
\text { S. A. de C.V. }\end{array}$ & $\begin{array}{c}\text { Ingeniero } \\
\text { José Ángel } \\
\text { Reyes Dávalos }\end{array}$ & $\begin{array}{c}\text { Director de Proyectos } \\
\text { de Corporación } \\
\text { Mexicana de } \\
\text { Investigación en } \\
\text { Materiales, S. A. de C.V V }\end{array}$ \\
\cline { 2 - 4 } & $\begin{array}{c}\text { Gobierno } \\
\text { Empresa: } \\
\text { Cluster de Energía } \\
\text { Coahuila A.C. }\end{array}$ & $\begin{array}{c}\text { Licenciada } \\
\text { Martha Laura } \\
\text { Carranza Aguayo }\end{array}$ & Directora adjunta \\
\hline
\end{tabular}

Fuente: elaboración propia, con la autorización de las personas mencionadas.

La metodología recomienda que el grupo discuta y acuerde el instrumento, sin embargo, debido a la dificultad para reunir a los miembros en un solo lugar, se decidió contactarlos por correo electrónico y se dio seguimiento vía telefónica. Respondieron ocho de los nueve especialistas: tres de Tamaulipas, tres de Nuevo León y dos de Coahuila, entidad donde no hubo respuesta del gobierno, lo que limitó en buena medida las estimaciones y los resultados. La aplicación se llevó a cabo de agosto a noviembre de 2015.

\section{El instrumento}

El instrumento empleado para recolectar la información y evaluar los efectos está integrado por 20 variables, relacionadas con la migración, actividades económicas, infraestructura y servicios públicos, patrimonio cultural y problemas sociales. Para evaluar dichas variables, cuenta con las secciones siguientes: a) dirección del efecto; b) intensidad del efecto; c) extensión del territorio; d) duración; e) tipo de efecto y f) probabilidad de ocurrencia.

La dirección del efecto (E) se refiere al carácter del impacto o signo, que puede ser positivo, negativo o neutral/sin cambio. Esta clasificación establece si la acción del proyecto es beneficiosa (signo positivo) o adversa (signo negativo) o permanece sin cambio (neutral) en la variable analizada. 
La intensidad del efecto (I) considera qué tan grave o fuerte puede ser la influencia del proyecto sobre la variable en cuestión (véase Figura 3).

Figura 3. Escala de intensidad ${ }^{4}$

\begin{tabular}{|c|c|}
\hline Respuesta & Valor \\
\hline Alta & 10 \\
\hline Media & 5 \\
\hline Baja & 2 \\
\hline
\end{tabular}

La extensión del territorio (Ex) es la superficie en la que inciden las acciones del proyecto, tanto directa como indirectamente, o el alcance global que se espera (véase Figura 4).

Figura 4. Escala de extensión

\begin{tabular}{|c|c|}
\hline Respuesta & Valor \\
\hline Regional & 10 \\
\hline Municipal & 5 \\
\hline Local & 2 \\
\hline
\end{tabular}

La duración (D) considera el tiempo que durará el efecto de la actividad del proyecto (véase Figura 5).

Figura 5. Escala de tiempo

\begin{tabular}{|c|c|}
\hline Respuesta & Valor \\
\hline Largo plazo & 10 \\
\hline Mediano plazo & 5 \\
\hline Corto plazo & 2 \\
\hline
\end{tabular}

4 Los valores se tomaron del estudio elaborado por la Food and Agriculture Organization (FAO 1995). 
El tipo de efecto o reversibilidad (R) se refiere a la capacidad del sistema para retornar a las condiciones originales una vez que terminan las actividades generadoras del impacto (véase Figura 6).

Figura 6. Escala de reversibilidad

\begin{tabular}{|c|c|}
\hline Respuesta & Valor \\
\hline Irreversible & 10 \\
\hline Parcialmente reversible & 5 \\
\hline Reversible & 2 \\
\hline
\end{tabular}

La probabilidad de ocurrencia (P) valora la probabilidad de que el efecto recaiga sobre la variable (véase Figura 7).

Figura 7. Escala de ocurrencia

\begin{tabular}{|c|c|}
\hline Respuesta & Valor \\
\hline Alta & 10 \\
\hline Media & 5 \\
\hline Baja & 2 \\
\hline
\end{tabular}

El índice de valoración o impacto (IV)

La construcción del índice de valoración o impacto se lleva a cabo en dos partes; en la primera se emplean las respuestas obtenidas de las variables I, Ex y D, y se calcula una nueva denominada magnitud (M), mediante la fórmula:

$$
M_{i}=\sum_{I=1}^{n}\left[\left(I_{i} * W_{I}\right)+\left(E x_{i} * W_{E x}\right)+\left(M_{i} * W_{D}\right)\right]
$$


en donde $\mathrm{M}_{\mathrm{i}}$ representa la magnitud obtenida para la variable $\mathrm{i}$, que en este caso puede ser cualquiera de las analizadas, por ejemplo: el patrimonio cultural; $W_{\mathrm{I}}, W_{\mathrm{Ex}}$ y $W_{\mathrm{D}}$ son ponderadores de la intensidad (I), la extensión (Ex) y la duración (D), que se usan para dar mayor peso a determinada variable y la suma de ellos debe ser igual a la unidad. ${ }^{5}$

En la segunda sección se toman las respuestas obtenidas de las variables R, P y M, y se calcula el índice de valoración o de impacto (IV) mediante la fórmula:

$$
I V_{i}=\prod_{l=1}^{n}\left[\left(R_{i}^{W_{R}} * R_{i}^{W P} * M_{i}^{W M}\right)\right]
$$

donde $I V_{\mathrm{i}}$ representa la medida obtenida para la variable $\mathrm{i}$, por ejemplo: el patrimonio cultural; $W_{\mathrm{R}}, W_{\mathrm{P}} \mathrm{y} W_{\mathrm{M}}$ son ponderadores de la reversibilidad (R), la probabilidad de ocurrencia (P) y la magnitud $(\mathrm{M})$; éstos se utilizan para dar mayor peso a una variable y la suma de ellos debe ser igual a la unidad, como en la primera parte. ${ }^{6} \mathrm{El} \mathrm{IV}$ puede tomar valores de 0 a 10 , donde 0 significa que el impacto sobre la variable es nulo o inexistente, mientras que un valor cercano a 10 , que es altamente probable. Y su efecto, ya sea positivo o negativo, dependerá del signo que presente dicho índice; para interpretar de mejor manera los resultados de éste se elaboró la Figura 8.

Figura 8. Jerarquización de impactos negativos

\begin{tabular}{|c|c|c|c|}
\hline Índice & $\begin{array}{c}\text { Nivel o } \\
\text { significado }\end{array}$ & Significado & Efectos negativos \\
\hline$>8.1$ & $\begin{array}{c}\text { Muy alto/ } \\
\text { crítico }\end{array}$ & $\begin{array}{c}\text { Probabilidad de ocurrencia } \\
\text { muy alta, con cambios } \\
\text { permanentese irreversibles }\end{array}$ & $\begin{array}{c}\text { Máxima atención. Medidas } \\
\text { preventivas para evitar } \\
\text { su manifestación }\end{array}$ \\
\hline $6.1-8.0$ & Alto & $\begin{array}{c}\text { Probabilidad de ocurrencia } \\
\text { alta, generan efectos graves, } \\
\text { negativos con consecuencias } \\
\text { de largo plazo }\end{array}$ & $\begin{array}{c}\text { Medidas mitigantes o } \\
\text { correctivas (de preferencia } \\
\text { las últimas). Normalmente } \\
\text { exigen monitoreo } \\
\text { o seguimiento }\end{array}$ \\
\hline
\end{tabular}

5 Aquí se emplearon los valores teóricos reportados por la FAO (1995): WI = 0.40; WEx = $0.40 ; W D=0.20$.

6 Aquí se emplearon los valores teóricos reportados por la FAO (1995): $W R=0.22 ; W P=$ $0.17 ; W \mathrm{M}=0.61$. 


\begin{tabular}{|c|c|c|c|}
\hline 4.1-6.0 & Medio & $\begin{array}{c}\text { Probabilidad de } \\
\text { ocurrencia moderada, } \\
\text { el impacto es mínimo } \\
\text { y no justifica la cancelación } \\
\text { del proyecto, pero } \\
\text { en combinación } \\
\text { con otros impactos puede } \\
\text { impedir su desarrollo }\end{array}$ & $\begin{array}{c}\text { Medidas preventivas, } \\
\text { que pueden sustituirse } \\
\text { por mitigantes, correctivas } \\
\text { o compensatorias cuando } \\
\text { el impacto se produzca, } \\
\text { si aquéllas resultaran costosas }\end{array}$ \\
\hline $2.1-4.0$ & Bajo & $\begin{array}{c}\text { Probabilidad de ocurrencia } \\
\text { baja o media, el impacto } \\
\text { es aceptable donde la } \\
\text { mitigación es deseable, } \\
\text { pero no esencial }\end{array}$ & $\begin{array}{c}\text { No se aplican medidas, } \\
\text { a menos que se trate } \\
\text { de áreas críticas o de } \\
\text { disposiciones económicas }\end{array}$ \\
\hline$\leq 2.0$ & Muy bajo & $\begin{array}{c}\text { Probabilidad } \\
\text { de ocurrencia muy baja }\end{array}$ & No se aplican medidas \\
\hline
\end{tabular}

Fuente: FAO (1995).

De esta manera, si una variable obtiene un valor de 3, con un signo negativo, su significancia es baja y es poco probable que en ella ocurra un efecto negativo, causado por la industria, a menos que se trate de áreas críticas o que los costos de los planes de mitigación sean bajos, es común que estos últimos no se apliquen. Por el contrario, si los valores son positivos, los cambios esperados en las variables se perciben como beneficiosos ante el desarrollo de la industria.

\section{Estimación del índice de valoración o de impacto}

La dificultad de integrar a todos los especialistas para evaluar, de manera consensuada, las variables propuestas implicó algunas modificaciones ad hoc en el procedimiento. En la dirección del efecto (E) se determinó emplear el signo que más se repite, así una variable podía presentar dos signos positivos más uno negativo y clasificarse como positivo; dos negativos más un positivo y etiquetarse como negativo o dos o tres valores de cero y clasificarse como neutral o sin efecto esperado en la variable de interés. Cuando se encontró un signo positivo, uno negativo y un cero, se tomó el del índice que presentó mayor valor numérico. Por último, se decidió emplear una etiqueta de inde- 
Figura 9. Estimación del índice de valoración o de impacto regional (Tamaulipas, Nuevo León y Coahuila)

\begin{tabular}{|c|c|c|c|}
\hline Variable & $\begin{array}{l}\text { Dirección } \\
\text { del efecto }\end{array}$ & Índice & Significancia \\
\hline Crecimiento de la población & Positivo & 5 & Medio \\
\hline Entrada de trabajadores de otros estados & Positivo & 6 & Medio \\
\hline Salud de la población & Neutral & 0 & Sin efecto \\
\hline Empleos & Positivo & 7 & Alto \\
\hline Ingresos & Positivo & 6 & Medio \\
\hline Cambio de uso de la tierra & Positivo & 4 & Bajo \\
\hline Cambio en la tenencia de la tierra & Positivo & 5 & Medio \\
\hline Unión de la gente & Positivo & 4 & Bajo \\
\hline La participación de la gente en las decisiones & Positivo & 4 & Bajo \\
\hline Las costumbres de la gente & Negativo & -3 & Bajo \\
\hline Patrimonio cultural & Neutral & 0 & Sin efecto \\
\hline Servicios públicos (agua, luz y drenaje) & Positivo & 7 & Alto \\
\hline Infraestructura (hoteles, casas y hospitales) & Positivo & 7 & Alto \\
\hline $\begin{array}{l}\text { Vías de comunicación } \\
\text { (carreteras, calles y caminos) }\end{array}$ & Positivo & 8 & Alto \\
\hline $\begin{array}{l}\text { Problemas sociales (delincuencia, prostitución, } \\
\text { vandalismo y drogadicción) }\end{array}$ & Positivo & 3 & Bajo \\
\hline Contaminación (agua, suelo y aire) & Negativo & -6 & Medio \\
\hline Servicios de protección civil & Neutral & 0 & Sin efecto \\
\hline Violencia & Positivo & 2 & Muy bajo \\
\hline Derechos humanos & Neutral & 0 & Sin efecto \\
\hline Disponibilidad de agua & Negativo & -6 & Medio \\
\hline
\end{tabular}

Fuente: elaboración propia, con datos de la encuesta. 
finición en los casos en los que se presentara un signo positivo, uno negativo, un cero y que el valor numérico fuese idéntico.

Para el cálculo del IV total, se emplearon las fórmulas descritas para las variables por especialista en los estados. Después se obtuvo el promedio total por cada una considerando sólo el valor absoluto de los números. De esta forma, si una variable presentaba un IV de -2 , -4, 0, el IV total se hacía de la manera siguiente: $[(2+4+0) / 3]$, lo cual daba como resultado 2 , con un signo negativo. Por el contrario, si otra variable tenía los mismos valores, pero dos con signo positivo, entonces el resultado numérico era el mismo, pero la dirección del efecto es considerada como positiva. Cuando el primer valor sea positivo y el segundo negativo, la dirección del efecto se considera negativa. Una vez que se estimó el IV para las variables en los estados, se elaboró el mismo procedimiento para calcular el IV para cada una (véase Figura 9).

\section{Discusión de los resultados}

La percepción del efecto regional de los especialistas sobre la población es positivo con una significancia media, como se aprecia en la Figura 9. Esto indica que las actividades de la industria traerán consigo el crecimiento de la población, con una probabilidad de ocurrencia media. Lo anterior va de acuerdo con lo esperado (Jacobsen y Parker 2014), ya que la expansión de este tipo de proyectos suele ir de la mano con un aumento demográfico, aunque ese no es el problema real per se de esta variable, sino más bien el incremento en la demanda de bienes, servicios e infraestructura que acarreará, y que podría convertirse en una dificultad para los gobiernos locales si no se cuenta con la infraestructura mínima.

Asimismo, se espera que la llegada de trabajadores de otros estados o regiones sea positiva para el lugar donde se instalen las compañías. Por un lado, esto puede incrementar la demanda de bienes y servicios de la localidad, así como reactivar la economía local y, por otro, la mano de obra de los trabajadores que llegan puede desplazar a la local y regional, sobre todo si no está capacitada para desempeñar los puestos requeridos. Esta situación podría detonar la delincuencia, la 
prostitución, el vandalismo, la drogadicción y la violencia en general, aunque de acuerdo con la respuesta de los expertos, se espera que el efecto sea contrario, en otras palabras, que los beneficios económicos sean lo suficientemente compensatorios como para reducir dichos problemas.

La salud pública se debe cuidar cuando se planean proyectos para el desarrollo, como la explotación de hidrocarburos y del gas shale; algunos de los problemas de salud asociados con estas actividades se relacionan con el aumento del estrés que la población puede llegar a experimentar (Jacquet 2009), así como un crecimiento de las enfermedades de trasmisión sexual, según el reporte emitido por la Food and Water Watch (2013) sobre Pensilvania, en Estados Unidos. Sin embargo, de acuerdo con los resultados obtenidos para el caso regional, no se esperan cambios significativos. No obstante, se debe poner atención a esta variable una vez que comience a funcionar la industria.

También se observa una significancia alta en la generación de empleo, pero con un impacto medio en los ingresos. Aunque los beneficios pudieran ser mayores como ocurrió en el condado estadounidense de Sublette, Wyoming, en donde el salario promedio de los trabajadores creció 104 por ciento, del año 2000 a 2007, mientras se mantenía el boom de la región (Jacquet 2009). No se debe olvidar que en el largo plazo las condiciones económicas y laborales cambian drásticamente. De manera que será importante cuidar que las condiciones de trabajo ofrecidas sean suficientes para alcanzar una mejor calidad de vida, que compense los costos sociales a los que estarán expuestos los trabajadores.

La puesta en marcha de planes, programas y proyectos en determinadas áreas puede incidir en la cohesión social, un factor muy importante, porque la mejor forma de frenar proyectos o impulsarlos depende mucho de la capacidad de organización de la sociedad. En el caso analizado, los resultados indican que la llegada de este tipo de industria al noreste de México será positiva para la unión de la gente, y su participación en la solución de los problemas que surjan de la explotación de recursos, aunque su intensidad o fuerza, así como su probabilidad de ocurrencia serán bajas. 
En lo referente a los usos y costumbres, el arribo de compañías a la región puede modificar el estilo de vida aparentemente tranquilo (típico de ciudades y áreas no desarrolladas), y volverlo más estresante (como en las urbes industriales). En este sentido, los expertos esperan un efecto negativo, con probabilidad de ocurrencia baja sobre el estilo de vida. Este signo negativo es muy importante porque ayuda a visualizar, de manera cualitativa, por dónde podrían aparecer las dificultades durante el curso del proyecto, para estar atentos e implementar acciones específicas para mitigar o evitar el daño. De acuerdo con la metodología descrita (signo y significancia), se espera que la explotación de gas shale modifique de manera negativa el estilo de vida en las localidades donde se ubiquen las compañías. Resultados que se correlacionan con los encontrados en los residentes más viejos del condado de Sublette, quienes señalan que las relaciones sociales de la comunidad han ido decayendo a raíz del boom energético, así como la satisfacción y la calidad de vida en general; además de su percepción de las repercusiones negativas de la industria sobre el medio ambiente (Jacquet 2009). Por lo anterior, será importante establecer medidas preventivas que fomenten las relaciones sociales entre los habitantes, para evitar la pérdida de los usos y costumbres.

El patrimonio cultural es otra variable que se clasificó como neutral o sin cambio por los especialistas. Aunque según la evidencia empírica sobre Estados Unidos, las actividades de explotación de hidrocarburos y del gas shale, mediante el fracking, pueden causar grandes problemas ambientales y culturales. Por lo que se deberá tener un cuidado especial para minimizar los riesgos que pongan en peligro el patrimonio cultural y natural.

La infraestructura general del lugar de la explotación será muy importante para favorecer un mejor crecimiento económico local y regional. Los resultados señalan que los expertos esperan un efecto positivo y con altas probabilidades de ocurrencia en cuanto a la creación de infraestructura para el abastecimiento de agua, luz y sistema de drenaje; la edificación de hoteles, casas, restaurantes y hospitales. El gran interrogante que se vislumbra es cómo se financiará y se solventará la deuda para las construcciones mencionadas, si no se tienen; además de resolver el destino de dicha infraestructura, en caso de que el ciclo de vida de la industria sea tan corto que no alcance a cubrir 
los pasivos adquiridos. Como en Pinedale, Wyomming, Estados Unidos, en donde se recibieron cerca de 20 millones de dólares por el petróleo y el gas en el año 2000, pero todos los ingresos se tuvieron que gastar en agua, alcantarillado e infraestructura vial (Ecosystem Research Group 2008). La literatura sobre las ciudades boom señala el gran perjuicio sobre las vías de comunicación, debido al tránsito de trasporte pesado, contrario a lo que los expertos esperan en esta región.

Para extraer gas shale o de lutitas se suele emplear la técnica de fracturación hidráulica o fracking, que requiere de grandes cantidades de agua; 98 por ciento del fluido inyectado es agua y 2 es de productos químicos. Si se considera todo el proceso, el consumo de agua aumenta de 10 a 30 por ciento en el lugar (Asamblea contra la Fractura Hidráulica 2011, 7), esto pone de manifiesto que de no contar con el agua suficiente, se podría generar desabasto en las ciudades aledañas a los campos de explotación, y el aumento de las presiones sociales de la comunidad. Sin duda, esta fue la preocupación que más externaron los especialistas, debido a que en la región hay sequía. De acuerdo con la Comisión Nacional del Agua (2014), la condición de Tamaulipas, Nuevo León y Coahuila, al final de la temporada seca de mayo de 2013, era de anormalmente seca a una de sequía severa, y en la parte fronteriza hasta de sequía excepcional. Además, la precipitación pluvial media de los tres estados es de $594 \mathrm{~mm}$, que si se compara con los $2095 \mathrm{~mm}$ de Tabasco, resulta muy baja. Aunado a las altas temperaturas de la región, el problema de abastecimiento físico de agua, generado por las condiciones naturales del medio ambiente se convierte en un riesgo latente.

Aunque se desconocen los efectos precisos de la extracción de gas shale, mediante la técnica del fracking, los resultados señalan que la contaminación del agua, el suelo y el aire también preocupa puesto que, de existir alguna contingencia o accidente, los mantos acuíferos se pueden contaminar, y la calidad y cantidad de agua disponible para otras actividades estaría en riesgo latente, lo que se debe tomar con toda seriedad en la regulación del sector. Por lo que habría que formular medidas preventivas, y exigir a las empresas planes específicos de acción para mitigar, corregir o compensar en caso de algún evento que contamine el lugar. 
Con la reforma energética actual, los propietarios de los terrenos donde se explore y exploten los recursos hidrocarburíferos podrán recibir el pago por usarlos. El cambio en la tenencia de la tierra puede generar beneficios económicos a los dueños actuales, algo que en la normativa anterior no era posible, y que también se ve reflejado en el signo positivo con una significancia media en dicha variable. Jacobsen y Parker (2014) señalaron este incremento en los ingresos de los terratenientes, debido a que las empresas deben pagar por el uso de suelo con su efecto multiplicador respectivo en la economía local. Sin embargo, los autores agregaron que en el largo plazo las cosas son muy distintas, se generaría una situación económica peor a la que prevalecía antes de iniciar la explotación. Por lo que el punto clave para generar los beneficios positivos estará en los contratos entre las compañías y los propietarios, y será necesario que los pagos por el uso de la tierra sean lo suficientemente buenos como para compensar su deterioro. Según los especialistas, en el cambio de uso de suelo el efecto positivo será bajo, contrario a lo que se podría esperar, debido a que se percibe como negativo por la pérdida de propiedades. Pero, de acuerdo con algunos entrevistados, las tierras en donde se pueden llevar a cabo este tipo de actividades son desérticas o no adecuadas para el cultivo, por lo cual quizá visualicen el efecto de manera positiva, aunque para especificarlo mejor se necesita una identificación puntual de las zonas y localidades que podrían experimentar este boom.

\section{Conclusiones}

Aquí se trabajó con la metodología de los criterios relevantes integrados para calcular un índice de valoración o de impacto sobre algunas variables como: crecimiento de la población, actividades económicas, infraestructura y servicios públicos, patrimonio cultural y contaminación, asociadas al desarrollo de la industria de hidrocarburos y en específico la explotación de gas shale. Para ello se aplicó un instrumento a informantes clave que representan, en alguna medida, la postura del gobierno, la academia y el sector empresarial en los tres estados que integran la cuenca de Burgos (Tamaulipas, Nuevo León y Coahuila). 
A pesar de que no todos los informantes respondieron, fue posible procesar los datos, aunque se reconoce la limitación que esto implicó para obtener mejores resultados. En general, en la región existe una sobrevaloración de los beneficios esperados por la reforma reciente, así como una subvaluación de los costos sociales en los que se incurrirá, debido a la llegada de las empresas de explotación. Los informantes esperan beneficios para los rubros relacionados con la construcción de infraestructura, servicios públicos y vías de comunicación, pero también que se agraven los problemas vinculados con la disponibilidad de agua en la región, porque la industria emplea grandes volúmenes para sus procesos, y en algún momento el consumo de los hogares pueda ponerse en riesgo; sobre todo en una región con sequías, altas temperaturas y poca precipitación pluvial. También se visualiza la contaminación del agua, aire y suelo, y se debe poner especial interés en regular y monitorear las repercusiones del fracking sobre estas variables, aunque la literatura no sea concluyente al respecto, ya que un problema de contaminación en cualquiera de ellas puede desencadenar daños irreparables para los ecosistemas y la salud de los habitantes, y poner en riesgo la sustentabilidad de las localidades.

Lo descrito ayuda a determinar el efecto que la industria pudiera traer sobre la zona, para estar preparados y aprovechar el boom económico. Para ello, la participación de los tres órdenes de gobierno será necesaria para crear políticas públicas que ayuden a incentivar los efectos positivos y a mitigar, evitar o compensar los negativos de la explotación. Hay que tener presente que el papel de las empresas será otro actor clave, y el daño que puedan causar a la sociedad dependerá de su responsabilidad social y ambiental.

Además, se encontró que Nuevo León, un estado netamente industrial y que depende en buena medida del gas para sus procesos productivos, es donde se esperan más efectos negativos. Se piensa que en Tamaulipas no habrá muchos cambios, y si ocurren serán positivos. En tanto que para Coahuila se visualiza que los beneficios de la explotación de los recursos naturales sean mayores. Esto se puede explicar en el contexto de la hipótesis de la curva medioambiental de Kuznets, en donde se supone que una economía que alcanza cierto crecimiento comienza a preocuparse y a cuidar el medio ambiente (Correa et al. 2005). En otras palabras, en dicha entidad ya cuentan 
con cierto grado de crecimiento económico y desarrollo, lo que les permite tener satisfechas sus necesidades y, en consecuencia, ya empiezan a pensar más en cuestiones ambientales. Caso contrario al de las otras dos, que buscan aprovechar la oportunidad de negocios para reactivar las actividades económicas.

\section{Bibliografía}

Asamblea contra la Fractura Hidráulica. 2011. La extracción de gas no convencional y la fractura hidráulica permisos en Burgos. http:/ / www.comimsa.com.mx/ (5 de junio de 2015).

Barbosa, Fabio. 2014. ¿Podemos obtener lecciones de la cuenca Marcellus? http://contralinea.info/archivo-revista/index. $\mathrm{php} / 2014 / 02 / 16 /$ podemos-obtener-lecciones-de-la-cuencamarcellus/ (7 de junio de 2015).

Barrueta, Eduardo. 2015. Gas de lutitas en la cuenca de Burgos. Energía y Debate. http://energiaadebate.com/gas-de-lutitas-en-la-cuencade-burgos/ (7 de junio de 2015).

Burdge, R, J. y F. Vanclay. 1996. Social impact assessment: a contribution to the state of the art series. Social Impact Assessment 14: 59-86.

Buroz, Eduardo. 1994. Métodos de evaluación de impactos. En II Curso de postgrado sobre Evaluación de Impactos Ambientales. Foro Latinoamericano de Ciencias Ambientales, La Plata.

Brown, Ralph, Shawn Dorius y Richard Krannich. 2005. The boombust-recovery cycle: dynamics of change in community satisfaction and social integration in Delta, Utah. Rural Sociology 70: 28-49.

Centro de Investigaciones Sociales. 2015. Reporte de diagnóstico general social, página 141. / http://www.impactosshalenoreste.uat. edu.mx/Documents/II.\%20DIAGNÓSTICO-GENERAL-SOCIAL. pdf (31 de enero de 2018). 
Comisión Nacional del Agua. 2014. Estadísticas del agua en México, edición 2014. Secretaría de Medio Ambiente y Recursos Naturales, México.

Correa Restrepo, Francisco, Andrés Vasco Ramírez y Catalina Pérez Montoya. 2005. La curva medioambiental de Kuznets: evidencia empírica para Colombia. Semestre Económico 8 (15): 13-30. http://revistas.udem.edu.co/index.php/economico/article/ view/1104/1075 (16 de marzo de 2017).

Davidson, Dona. 1979. Overview of the boomtown phenomenon and its effect on women and minorities. U.S. Commission on Civil Rights Energy Resource Development Washington, D.C.: U.S. Government Printing Office.

DOF. 2014. Ley de Hidrocarburos. 11 de agosto.

Ecosystem Research Group. 2008. Sublette county socioeconomic impact study Sublette county, Wyoming. http://sublette-se.org/ files/SubletteProfile11Feb08.pdf (17 de marzo de 2017).

Energy International Administration. 2013. EIA/ARI World Shale Gas and Shale Oil Resource Assessment. http://www.adv-res.com/ pdf/A_EIA_ARI_2013\%20World\%20Shale $\% 20 \mathrm{Gas} \% 20$ and $\% 20$ Shale\%20Oil\%20Resource\%20Assessment.pdf (1 de junio de 2015).

Flores, Daniel. 2014. Comentarios sobre la reforma energética. Boletín mensual del Centro de Investigaciones Económicas. Facultad de Economía, Universidad Autónoma de Nuevo León.

FAO. 1995. Impacto ambiental de las prácticas de cosecha forestal y construcción de caminos en bosques nativos siempre verdes de la X Región de Chile. Estudio monográfico. http://www.fao.org/ docrep/V9727S/V9727S00.htm (4 de diciembre de 2015). 
Food and Water Watch. 2013. The social cost of fracking: a Pennsyilvania case study. https://www.foodandwaterwatch.org/reports/ the-social-costs-of-fracking/ (5 de junio de 2015).

TheWall Street Journal. 2008. Gas producers rush to Pennsylvania: promising results there spur investment. 2 de abril. https://www.wsj. com/articles/SB120709326316581793 (16 de marzo de 2017). Horton, Paul y Gerald Leslie. 1955. The sociology of social problems. Nueva York: Appiclon-Century-Cropts.

Interorganizational Committee on Guidelines and Principles for Social Impact Assessment. 1995. Guidelines and principles for social impact assessment. Environ Impact Assess Review 15: 1 I-43.

Jacquet, Jeffrey. 2009. Energy boomtowns and natural gas: implications for Marcellus shale local governments and rural communities. Paper 43. Northeast Regional Center for Rural Development, Pennstate. http://nercrd.psu.edu/Publications/rdppapers/rdp43. pdf (1 de mayo de 2015).

Jacobsen, Grant y Dominic Parker. 2014. The economic aftermath of resource booms: evidence from boomtowns in the American west. The Economic Journal 126: 1092-1128. Doi: 10.1111/ecoj.12173

Kohrs, Eldean. 1974. Social consequences of boom growth in Wyoming. Ponencia presentada en Rocky Mountain American Association of the Advancement of Science Meeting, Laramie, Wyoming. http://www.sublettewyo.com/ArchiveCenter/ViewFile/Item/97 (1 de mayo de 2015).

MacPherson, Matthew. 2004. Guía para la realización de las evaluaciones de impacto social dentro del proceso de evaluación de impacto ambiental. Secretaría de Estado de Medio Ambiente y Recursos Naturales. Santo Domingo, República Dominicana. http://www.ambiente.gob.do/Transparencia/Legal/Guia/Guia-para-realizacionEvaluaciones-Impacto-Social.pdf (3 de mayo de 2015). 
Muller, Richard y Elizabeth Muller. 2013. Why every serious environmentalist should favour fracking. Centre for Policy Studies. http://www.cps.org.uk/files/reports/original/131202135150WhyEverySeriousEnvironmentalistShouldFavourFracking.pdf (12 de marzo de 2017).

Schafft, Kai A., Borlu Yetkin y Glenna Leland. 2013. The relationship between Marcellus shale gas development in Pennsylvania and local perceptions of risk and opportunity. Rural Sociology 78: 143-66.

Secretaría de Energía. 2014. Prospectiva del gas natural y gas LP 2014-2028. https://www.gob.mx/cms/uploads/attachment/ file/352/Prospectiva-GasNaturalGasLP-2014.pdf (16 de mayo de 2016).

SEMARNAT. 2008. Ordenamiento ecológico: región cuenca de Burgos. México. http://www.semarnat.gob.mx/archivosanteriores/ temas/ordenamientoecologico/Documents/documentos_burgos/zip/resumen_ejecutivo_mar_08_2.pdf(20 de julio de 2015).

Suárez, Francisco M. 1989. Problemas sociales y problemas de programas sociales masivos. Trabajo presentado en el Seminario sobre programas sociales masivos, organizado por el Centro Interamericano para el Desarrollo. Social/Organización de Estados Americanos y el Instituto Latinoamericano y del Caribe de Planificación Económica y Social, San José, Costa Rica. http://archivo.cepal. org/pdfs/1989/S8900328.pdf (20 de julio de 2015).

Theodori, Gene. 2012. Public perception of the natural gas industry: data from the Barnett Shale. Energy Sources, part B 7: 275-281.

Theodori, Gene y Douglas Jackson-Smith. 2010. Public perception of the oil and gas industry: the good, the bad and the ugly. Ponencia presentada en la Society of Petroleum Engineers Annual Technical Conference and Exhibition, Florencia. 
Vanclay, Frank. 2003. International principles for social impact assessment. Impact Assessment \& Project Appraisal 21 (1): 5-11. Doi: http:// dx.doi.org/10.3152/147154603781766491

Vanclay, Frank. 2002. Conceptualising social impacts. Environmental Impact Assessment Review 22 (3): 183-211. 